Ambiente \& Água - An Interdisciplinary Journal of Applied Science
ISSN 1980-993X - doi:10.4136/1980-993X
www.ambi-agua.net
E-mail: ambi.agua@gmail.com

\title{
Reviewers of Volume 10, 2015 of Revista Ambiente \& Água
}

\section{Masthead Section}

\author{
doi:10.4136/ambi-agua.1792 \\ Getulio Teixeira Batista \\ Editor of Revista Ambiente \& Água \\ e-mail: ambi.agua@gmail.com
}

The following list includes the names and affiliations of all reviewers that evaluated the submissions processed for Volume 10, 2015 of Revista Ambiente \& Água, including manuscripts that were accepted and published in one of the four annual issues and also manuscripts submitted and rejected in 2015 (Table 1). It may therefore include the names of reviewers that completed their evaluations between September, 2014 and October, 2015. Several of the reviewers evaluated more than one manuscript.

We are deeply indebted to all of them that voluntarily and anonymously spent their precious time to support the journal, and contributed immensely to the quality of the published articles. We gratefully acknowledge their professional work and outstanding product.

Table 1. Ad Hoc Peer Reviewers that evaluated submissions for the 2015 edition of Revista Ambiente \& Água, Volume 10, n. 1, 2, 3 and 4, 2015.

Abel Talabi

Ademir Kleber de Oliveira

Adriane Martins de Freitas

Alberto Adriano Cavalheiro

Aldo Pacheco Ferreira

Alessandra Cristina Silva Valentim

Alexandra Sofia Baptista Vicente

Baeta

Alexandre Lioi Nascentes

Aline Maria Meiguins de Lima

Aline Sueli de Lima Rodrigues

Ana Cristina Gobbo César

Ana Lucia Suriani Affonso
Ekiti State University, Ekiti State University, Ado Ekiti, Nigeria

Universidade Anhanguera (UNIDERP)

Universidade Tecnológica Federal do Paraná (UTFPR)

Universidade Estadual de Mato Grosso do Sul (UEMS)

Escola Nacional de Saúde Pública (FIOCRUZ)

Universidade Federal do Recôncavo da Bahia (UFRB)

Marine and Environmental Sciences Centre, Faculty of Sciences and Technology, Univ. of Coimbra, Portugal

Universidade Federal Rural do Rio de Janeiro (UFRRJ)

Universidade Federal do Pará (UFPA)

Instituto Federal Goiano (IF Goiano - Câmpus Urutaí)

Instituto Federal de São Paulo (IFSP)

Universidade Estadual do Centro-Oeste (UNICENTRO) 
André Felippe Nunes Freitas

Andre Luis Teixeira Fernandes

André Monteiro Costa

André Quintão de Almeida

André Ricardo de Araújo Lima

Andrea Giuseppe Capodaglio

Andrea Nunes Vaz Pedroso

Andreia Maria Anunciação Gomes

Anne Hélène Fostier

Antonio Cezar Leal

Antonio Laverde Junior

Antônio Pereira Magalhães Junio

Armando Augusto Henriques Vieira

Caio Fernando Gromboni

Carla Cristine Müller Cestari

Carlos Alexandre Borges Garcia

Carlos Alexandre Rey Matias

Carlos Alexandre Santos Querino

Carlos Elizio Cotrim

Carlos Frederico Bernardo Loureiro

Catarinie Diniz Pereira

Célia Alves Souza

César Augusto Moreira

Cilia Leonor Fuentes Piedrahita

Ciro Alberto de Oliveira Ribeiro

Claudia Albuquerque Linhares

Cláudia Maria Luz Lapa Teixeira

Claudinei Fonseca Souza

Claudio Fernando Mahler

Claudio José Von Zuben
Universidade Federal Rural do Rio de Janeiro (UFRRJ)

Universidade de Uberaba (UNIUBE)

Fundação Oswaldo Cruz (FIOCRUZ)

Universidade Federal de Sergipe (UFS)

Universidade Federal de Pernambuco (UFPE)

Università degli Studi di Pavia, Pavia, Itália

Instituto de Botânica (IBT)

Universidade Federal de Itajubá (UNIFEI)

Universidade Estadual de Campinas (UNICAMP)

Univ. Estadual Paulista Júlio de Mesquita Filho (UNESP)

Universidade Tecnológica Federal do Paraná (UTFPR)

Universidade Federal de Minas Gerais (UFMG)

Universidade Federal de São Carlos (UFSCAR)

Instituto Federal da Bahia (IFBA)

Companhia Riograndense de Saneamento (CORSAN)

Universidade Federal de Sergipe (UFS)

Universidade Federal Rural do Rio de Janeiro (UFRRJ)

Centro Gestor e Operacional do Sistema de Proteção da Amazônia (CENSIPAM)

Instituto Federal de Educação Ciência e Tecnologia Baiano (IFBAIANO)

Universidade Federal do Rio de Janeiro (UFRJ)

Universidade Estadual de Campinas (UNICAMP)

Universidade do Estado de Mato Grosso (UNEMAT)

Univ. Estadual Paulista Júlio de Mesquita Filho (UNESP)

Universidad Nacional de Colombia, Bogotá, Colombia

Universidade Federal do Paraná (UFPR)

Instituto Nacional de Pesquisas Espaciais (INPE)

Instituto Nacional de Tecnologia (INT)

Universidade Federal de São Carlos (UFSCAR)

Universidade Federal do Rio de Janeiro (UFRJ)

Univ. Estadual Paulista Júlio de Mesquita Filho (UNESP) 


\section{Claudio Marcio Souza}

Claudio Campos Montenegro

Cleber Antonio Lindino

Clovis Antonio Rodrigues

Conrado de Moraes Rudorff

Daiana Cardoso de Oliveira

Daniel Vidal Perez

Danielle Palma de Oliveira

Danilo Paulúcio da Silva

Delvio Sandri

Diego de Souza Sardinha

Diego Rodrigues Macedo

Eden Albuquerque Junior

Ederio Dino Bidoia

Edison Barbieri

Eduardo Rodrigues Viana de Lima

Eline Simões Gonçalves

Elmo Rodrigues da Silva

Elvis Vasquez Rimachi

Érika Andressa da Silva

Fabiana Valéria da Fonseca

Fábio Kummrow

Fábio Marcelo Breunig

Fabio de Oliveira Sanches

Fabrício Berton Zanchi

Fernando França da Cunha

Flávia Maria Galizoni

Flavia Mariani Barros

Flávio Rubens Lapolli

Gabriel Constantino Blain
Universidade Federal dos Vales do Jequitinhonha e Mucuri (UFVJM)

Universidade Federal de Lavras (UFLA)

Universidade Estadual do Oeste do Paraná (UNIOESTE)

Universidade do Vale do Itajaí (UNIVALI)

Centro Nacional de Monitoramento e Alertas de Desastres Naturais (CEMADEN)

Universidade Federal de Santa Catarina (UFSC)

Empresa Brasileira de Pesquisa Agropecuária (EMBRAPA)

Fac. de C. Farmacêuticas de Rib. Preto (FCFRP/USP)

Universidade Estadual do Sudoeste da Bahia (UESB)

Universidade de Brasília (UnB)

Universidade Federal de Alfenas (UNIFAL)

Fundação Instituto Brasileiro de Geografia e Estatística (IBGE)

Instituto de Tecnologia de Pernambuco (ITEP)

Univ. Estadual Paulista Júlio de Mesquita Filho (UNESP)

Instituto de Pesca (IP)

Universidade Federal da Paraíba (UFPB)

Fundação Oswaldo Cruz (FIOCRUZ)

Universidade do Estado do Rio de Janeiro (UERJ)

Instituto Nacional da Amazônia (INPA)

Universidade Federal de Lavras (UFLA)

Universidade Federal do Rio de Janeiro (UFRJ)

Universidade Federal de São Paulo (UNIFESP)

Universidade Federal de Santa Maria (UFSM)

Universidade Federal da Fronteira Sul (UFFS)

Universidade Federal do Amazonas (UFAM)

Universidade Federal dos Vales do Jequitinhonha e Mucuri (UFVJM)

Universidade Federal de Minas Gerais (UFMG)

Universidade Estadual do Sudoeste da Bahia (UESB)

Universidade Federal de Santa Catarina (UFSC)

Instituto Agronômico de Campinas (IAC) 
Gabriela Vázquez Hurtado

Gannabathula Sree Vani

Geison Jader Mello

Gerson Araujo de Medeiros

Gesinaldo Ataíde Cândido

Getulio Teixeira Batista

Gilberto Chohaku Sediyama

Gilvan Sampaio de Oliveira

Giordano Urbini

Giovani de Oliveira Garcia

Gleyci Aparecida Oliveira Moser

Gordon Tami Amangabara

Guilherme Luiz Dotto

Guilherme Malafaia

Guilherme Viana de Alencar

Henry Louis Spach

Honoria de Fatima Gorgulho

Hugo Alexandre Soares Guedes

Hycienth Nwankwoala

Irajá do Nascimento Filho

Iria Fernandes Vendrame

Irineu Bianchini Júnior

Isabel Cristina de Barros Trannin

Jácomo Divino Borges

Jaime Lopes da Mota Oliveira

Jair Max Furtunato Maia

Jairo Osvaldo Cazetta

João Carlos Costa Guimarães

Jonas Teixeira Nery
Universidad Veracruzana, Instituto de Ecología (INECOL), Veracruz, México

Universidade de Taubaté (UNITAU)

Instituto Federal de Mato Grosso (IFMT)

Univ. Estadual Paulista Júlio de Mesquita Filho (UNESP)

Universidade Federal de Campina Grande (UFCG)

Universidade de Taubaté (UNITAU)

Universidade Federal de Viçosa (UFV)

Instituto Nacional de Pesquisas Espaciais (INPE)

Università Degli Studi dell'Insubria, Varese, Italia

Centro de C. Agrárias da Univ. Fed. do ES (CCAUFES)

Universidade do Estado do Rio de Janeiro (UERJ)

Federal University of Technology, Owerri, Nigeria

Universidade Federal de Santa Maria (UFSM)

Instituto Federal Goiano (IF Goiano - Câmpus Urutaí)

Inst. Brasileiro do Meio Ambiente e dos Recursos Naturais Renováveis (IBAMA)

Universidade Federal do Paraná (UFPR)

Universidade Federal de São João Del-Rei (UFSJ)

Universidade Federal de Pelotas (UFPEL)

University of Port-Harcourt, Port Harcourt, Rivers State, Nigeria

Universidade de Caxias do Sul (UCS)

Instituto Tecnológico de Aeronáutica (ITA)

Universidade Federal de São Carlos (UFSCar)

Univ. Estadual Paulista Júlio de Mesquita Filho (UNESP)

Universidade Federal de Goiás (UFG)

Departamento de Saneamento e Saúde Ambiental (ENSP/FIOCRUZ)

Universidade do Estado do Amazonas (UEA)

Univ. Estadual Paulista Júlio de Mesquita Filho (UNESP)

Universidade Federal de Itajubá (UNIFEI)

Unidade Diferenciada Ourinhos (UNESP) 


\section{Joost van Haren}

Jorge Armando Guevara Gil

Jorge Luiz Rodrigues Filho

José Antonio Rodrigues de Souza

José Augusto de Lollo

José Carlos Mierzwa

José Cleidimário Araújo Leite

José Dias Corrêa Junior

José Marinaldo Gleriani

José Luis Ibarra-Montoya

José Luiz Silvério da Silva

José Max Barbosa Oliveira Jr.

Josely Dantas Fernandes

Josino Costa Moreira

Julio Cesar Raposo de Almeida

Julio Pascale Palhares

Julio Vieira Neto

Karime Rita de Souza Bentes

Katia Canil

Kaveh Madani

Kelly das Graças Fernandes Dantas

Kettrin Farias Bem Maracajá

Lincoln Eloi de Araújo

Lourival Ferreira Cavalcante

Luciano dos Santos Rodrigues

Luisa Elizabeth Delgado Isasi

Luiz Augusto do Amaral

Luiz Augusto Manfré

Luiz Drude de Lacerda
The University of Arizona, Tucson, Arizona, US

Pontifica Universidad Católica del Perú, San Miguel, Lima Perú

Universidade do Estado de Santa Catarina (UDESC)

Instituto Federal Goiano (IF Goiano - Câmpus Urutaí)

Univ. Estadual Paulista Júlio de Mesquita Filho (UNESP)

Universidade de São Paulo (USP)

Universidade Federal de Campina Grande (UFCG)

Universidade Federal de Minas Gerais (UFMG)

Universidade Federal de Viçosa (UFV)

Instituto Tecnológico de Estudios Superiores de Moterrey, México

Universidade Federal de Santa Maria (UFSM)

Universidade do Estado de Mato Grosso (UEMG)

Universidade Estadual da Paraíba (UEPB)

Fundação Oswaldo Cruz (FIOCRUZ)

Universidade de Taubaté (UNITAU)

Pecuária Sudeste (EMBRAPA)

Universidade Federal Fluminense (UFF)

Universidade Federal do Amazonas (UFAM)

Universidade Federal do ABC (UFABC)

Imperial College London, South Kensington Campus, London, UK

Universidade Federal do Pará (UFPA)

Universidade Federal do Rio Grande do Norte (UFRN)

Universidade Federal da Paraíba (UFPB)

Universidade Federal de Campina Grande (UFCG)

Universidade Federal de Minas Gerais (UFMG)

Universidad de Chile, Fundacion Centro Transdisciplinario de Estudios Fes-Sistemicos (CTF-FES), Santiago, Chile

Univ. Estadual Paulista Júlio de Mesquita Filho (UNESP)

Cargill, Incorporated, Agribusiness Intelligence, MN, US

Universidade Federal do Ceará (UFC) 
Luiz Ermindo Cavallet

Luiz Fernando Scheibe

Luiz Roberto Santos Moraes

Magali Christe Cammarota

Magali Rezende Gouvea Meireles

Manuel de Jesus Flores Montes

Marcel Fantin

Marcelo De Julio

Marcelo Henrique Otenio

Marcelo Sandin Dourado

Marcelo Tavares Gurgel

Marcia Terezinha Zilli

Marcio Rossi

Marcos Antonio Gomes

Marcos Moreira de Barros Salomão

Maria Cristina Borba Braga

Maria do Carmo Martins Sobral

Maria Gizelda de Oliveira Tavares

Maria Lourdes Souza Santos

Maria Rosaria Boni

Maria Teresa Calvão Rodrigues

Marluce Martins Martins de Aguiar

Marselle Bevilacqua Amadio

Marta Margarete Cestari

Masato Kobiyama

Massimo Raboni

Matheus Fonseca Durães

Mathias Alberto Schramm

Maurea Nicoletti Flynn
Universidade Estadual do Paraná (UNESPAR)

Universidade Federal de Santa Catarina (UFSC)

Universidade Federal da Bahia (UFBA)

Universidade Federal do Rio de Janeiro (UFRJ)

Pontifícia Universidade Católica de Minas Gerais (PUC)

Universidade Federal de Pernambuco (UFPE)

Instituto de Arquitetura e Urbanismo (USP)

Instituto Tecnológico de Aeronáutica (ITA)

Empresa Brasileira de Pesquisa Agropecuária (EMBRAPA)

Universidade Federal do Paraná (UFPR)

Universidade Federal Rural do Semi-Árido(UFERSA)

University of California, , Santa Barabra, California, US

Instituto Florestal do Estado de São Paulo (IF)

Universidade Federal de Viçosa (UFV)

Universidade Estadual do Norte Fluminense Darcy Ribeiro (UENF)

Universidade Federal do Paraná (UFPR)

Technische Universität Berlin, Berlin, Deutschland

Universidade Federal de Goiás (UFG)

Universidade Federal Rural da Amazônia (UFRA)

University of Rome "La Sapienza", Roma, Italia

Universidade Nova de Lisboa (UNL), Lisboa, Portugal

Instituto Federal de Educação Ciência e Tecnologia do Espírito Santo (IFES)

Centro Universitário Senac (SENAC/SP)

Universidade Federal do Paraná (UFPR)

Universidade Federal de Santa Catarina (UFSC)

University LIUC, Varese, Italia

Universidade Federal de Lavras (UFLA)

Instituto Federal de Educação Ciência e Tecnologia de Santa Catarina (IF/SC)

EcoAdvisor Associados, São Paulo, SP 
Mauricio José Borges

Mauricio Luiz Sens

Mesfin Mergia Mekonnen

Michele Morais Oliveira Pereira

Miguel Mansur Aisse

Mônica Luisa Kuhlmann

Monica Maria Pereira da Silva

Montse Meneses

Nádia Hortense Torres

Natalino Perovano Filho

Nei Kavaguichi Leite

Nelson Wellausen Dias

Nildo da Silva Dias

Núbia Natália de Brito

Olga Venimar de Oliveira Gomes

Osmar Gustavo Wöhl Coelho

Osvaldo Luiz Gonçalves Quelhas

Otidene Rossiter Sa da Rocha

Paulo Cesar de Jesus

Paulo Cesar Rocha

Paulo Cesar Sentelhas

Paulo Henrique Caramori

Pedro Daniel da Cunha Kemerich

Priscila Fernanda Campos de Menezes

Rafael Oliveira Batista

Regina Peralta Moreira

Reinaldo G Bastos

Reinaldo Vargas

Renata Stábile Amais

Renata de Souza Leão Martins
Instituto Taquaritinguense de Ensino Superior, Taquaritinga, SP

Universidade Federal de Santa Catarina (UFSC)

University of Twente, Enschede, The Netherlands

Universidade Federal de Viçosa (UFV)

Universidade Federal do Paraná (UFPR)

Companhia Ambiental do Estado de São Paulo (CETESB)

Universidade Estadual da Paraíba (UFPB)

Universitat Autonoma de Barcelona (UAB), Bellaterra, Barcelona, Espanha

Instituto de Tecnologia e Pesquisa (ITP)

Universidade Estadual do Sudoeste da Bahia (UESB)

Universidade Federal de Santa Catarina (UFSC)

Instituto Brasileiro de Geografia e Estatística (IBGE)

Universidade Federal Rural do Semi-Árido (UFERSA)

Universidade Federal de Goiás (UFG)

Universidade Federal Fluminense (UFF)

Universidade do Vale do Rio dos Sinos (UNISINOS)

Universidade Federal Fluminense (UFF)

Universidade Federal de Pernambuco (UFPE)

Universidade Federal do Rio de Janeiro (UFRJ)

Univ. Estadual Paulista Júlio de Mesquita Filho (UNESP)

Universidade de São Paulo (ESALQ/USP)

Instituto Agronômico do Paraná (IAPAR)

Universidade Federal de Santa Maria (UFSM/CESNORS)

Instituto de Física e Química de São Carlos (USP)

Universidade Federal Rural do Semi-Árido (UFERSA)

Universidade Federal de Santa Catarina (UFSC)

Universidade Federal de São Carlos (UFSCAR)

Universidade de Guarulhos (UNG)

Centro de Energia Nuclear na Agricultura (CENA/USP)

Instituto de Energia e Ambiente (IEE) 
Renato Farias do Valle Junior

Renato Gavasci

Renato Igor da silva Alves

Ricardo dos Santos Coelho

Rita de Cássia Colman Simões

Roberto Avelino Cecílio

Roberto Moraes Lima Silveira

Rodolfo Carapelli

Rogério Caetano da Costa

Ronaldo Borges Barthem

Ronaldo de Almeida

Ronaldo Fia

Ronaldo Teixeira Pelegrini

Rosana Icassatti Corazza

Rose Maria Duda

Rute Isabel Costa Pinto

Sandro Geraldo de Castro Britto

Selma Gomes Ferreira Leite

Selma Simões de Castro

Sérgio Marcos Sanches

Sergio Nascimento Duarte

Sergio Salinas

Sigrid Neumann Leitão

Silvia Mattos Nascimento

Silvio Bueno Pereira

Simone Andrea Pozza

Simone Bittencourt

Simone Maria Ribas Vendramel

Sueli Yoshinaga Pereira
Instituto Federal de Educação Ciência e Tecnologia do Triângulo Mineiro (IFTM)

University of Rome "Tor Vergata", Roma, Italia

Universidade de São Paulo (USP)

Instituto Federal de São Paulo (IFSP)

Universidade Federal Fluminense (UFF)

Universidade Federal do Espírito Santo (UFES)

Universidade Federal de Mato Grosso (UFMT)

Universidade Federal do Rio Grande (FURG)

Univ. Estadual Paulista Júlio de Mesquita Filho (UNESP)

Museu Paraense Emílio Goeldi (MPEG)

Universidade Federal do Amazonas (UFAM)

Universidade Federal de Lavras (UFLA)

Universidade Federal de São Carlos (UFSCAR)

Faculdades de Campinas (FACAMP)

Univ. Estadual Paulista Júlio de Mesquita Filho (UNESP)

Marine and Environmental Research Centre (IMAR-CMA), Coimbra, Portugal

Univ. Estadual Paulista Júlio de Mesquita Filho (UNESP) Laboratório de Biologia e Ecologia de Peixes, Botucatu

Universidade Federal do Rio de Janeiro (UFRJ)

Universidade Federal de Goiás (UFG)

Instituto Federal de Educação Ciência e Tecnologia do Triângulo Mineiro (IFTM)

Universidade de São Paulo (ESALQ-USP)

Universidad de Zaragoza, Zaragoza, Espanha

Universidade Federal de Pernambuco (UFPE)

Universidade Federal do Estado do Rio de Janeiro (UNIRIO)

Universidade Federal de Viçosa (UFV)

Universidade Estadual de Campinas (UNICAMP)

Companhia de Saneamento do Paraná (SANEPAR)

Instituto Federal de Educação Ciência e Tecnologia do Rio de Janeiro (IFRJ)

Universidade Estadual de Campinas (UNICAMP) 
Tamara Maria Gomes

Tânia Regina Kinasz de Oliveira

Teodorico Alves Sobrinho

Teresa Cristina Brazil de Paiva

Teresa Maria Reyna

Toni Jefferson Lopes

Tulio Assunção Pires Ribeiro

Valéria Peixoto Borges

Valérie Genty Pot

Vandré Barbosa Brião

Vanessa Empinotti

Vincenzo Torretta

Wagner Walker de Albuquerque Alves

Wallisson da Silva Freitas

Walter de Paula Lima

Welber Senteio Smith

Willames de Albuquerque Soares

Zagabathuni Venkata Panchakshari Murthy
Universidade de São Paulo (USP)

Universidade Federal de Mato Grosso (UFMT)

Universidade Federal de Mato Grosso do Sul (UFMS)

Universidade de São Paulo (USP)

Universidad Nacional de Córdoba, Córdoba, Argentina

Universidade Federal do Rio Grande do Sul (UFRGS)

Universidade Estadual de Campinas (UNICAMP)

Universidade Federal da Paraíba (UFPB)

Institut National de la Recherche Agronomique (INRA), Marrocos

Universidade de Passo Fundo (UPF)

Universidade de São Paulo (USP)

University of Insubria, Varese, Italia

Universidade Federal de Rondônia (UNIR)

Instituto Federal de Educação Ciência e Tecnologia do Espírito Santo (IFES)

Universidade de São Paulo (USP)

Universidade Paulista (UNIP)

Universidade de Pernambuco (UPE)

S. V. National Institute of Technology, Gujarat, India

Note: 1) Some of these reviewers evaluated more than one manuscript; 2) This list includes reviewers that evaluated submissions that were not accepted for publication, in addition to the ones that were published in 2015.

As seen in Table 2, 27 reviewers are from international institutions abroad. This is an indication that the journal is gaining international acceptance and inclusion.

Table 2. Number and type of institutions of reviewers that contributed to Volume 10 of 2015.

\begin{tabular}{|c|c|c|c|c|c|c|}
\hline \multicolumn{5}{|c|}{ From Brazil } & \multirow{2}{*}{$\begin{array}{c}\text { From abroad } \\
\text { Reviewers from } \\
\text { international Institutions }\end{array}$} & \multirow[b]{2}{*}{ Tota } \\
\hline $\begin{array}{c}\text { Federal } \\
\text { Universities }\end{array}$ & $\begin{array}{c}\text { State } \\
\text { Universities }\end{array}$ & $\begin{array}{c}\text { Federal } \\
\text { Institutions }\end{array}$ & $\begin{array}{c}\text { National } \\
\text { private } \\
\text { Universities } \\
\end{array}$ & $\begin{array}{c}\text { Municipal } \\
\text { Universities }\end{array}$ & & \\
\hline 101 & 49 & 48 & 8 & 3 & 27 & 236 \\
\hline
\end{tabular}

